\title{
New perturbation theory for nonstationary anharmonic oscillator
}

\author{
Alexander V. Bogdanov and Ashot S. Gevorkyan \\ Institute for High-Performance Computing and Data Bases \\ P/O Box, 71, St-Petersburg, 194291, Russia
}

\begin{abstract}
The new perturbation theory for the problem of nonstationary anharmonic oscillator with polynomial nonstationary perturbation is proposed. As a zero order approximation the exact wave function of harmonic oscillator with variable frequency in external field is used. Based on some intrinsic properties of unperturbed wave function the variational-iterational method is proposed, that make it possible to correct both the amplitude and the phase of wave function. As an application the first order correction are proposed both for wave function and $S$-matrix elements for asymmetric perturbation potential of type $V(x, \tau)=\alpha(\tau) x^{3}+\beta(\tau) x^{4}$. The transition amplitude "ground state - ground state" $W_{00}(\lambda ; \rho)$ is analyzed in detail depending on perturbation parameter $\lambda$ (including strong coupling region $\lambda$ $\sim 1$ ) and one-dimensional refraction coefficient $\rho$.
\end{abstract}

\section{Introduction}

Every successful effort in investigation of some physical problem in strong interaction region usually brings new insight in behavior of corresponding system. Thus, the outstanding results, obtained in the solution of one-dimensional model problems in quantum field theory, molecular and solid-state physics [1 - 3] are of great interest. But the investigation becomes much more complicated when many-dimensional problems are approached. In such situation contradictory results are possible even in calculations of the system eigenenergy [4] .

The situation becomes even more dramatic while dealing with nonstationary problems with eigenfunctions basis, changing in time. The most important example of it is given by model of two-dimensional scattering problem, that is called collinear model for rearrangement [5]. It is one of the simplest and still realistic descriptions of the three-body reaction of $A+(B, C)_{n} \rightarrow(A, B)_{m}+C$ type.

This problem can be of principle importance both for the practical applications in the theory of chemical reaction for moderate energies and for illuminating the mechanisms of rearrangement in multichannel scattering theory. It can be also regarded as a good testbed for application of nonperturbative method in realistic few body problem with all reaction channels involved.

In our previous paper [6] it was shown, that in the limit $\hbar \rightarrow 0$ this problem is effectively reduced to that for one-dimensional anharmonic oscillators with the frequency 
$\Omega(\tau)$, changing in the field of external force $F(\tau)$. Numerous quantum-chemical calculations of the potential energy surface for most popular three-particle systems have shown strong anharmonism in normal coordinate even for principle quantum number as low, as $n \geq 3 \div 4$.

Here we propose the method, that seems to be a natural development of the ideas of stationary perturbation theory of [3] for the case of nonstationary coefficients of a zero-order equation. The method is of variational/iteration type and is essentially backed by main ideas of singular perturbation theory - one can expand only such parts of the solution, that do not bring singularities, and if one can not find pattern for singular part of the solution it is possible to expand it in the exponential.

Our final goal would be derivation of the scattering matrix representation, that makes it possible the simultaneous computation of the probabilities for all pertinent scattering mechanisms, taking into account close coupling of many channels, resonance effects and chaotic behavior in intermediate state.

The perturbation theory proposed might be especially interesting for calculation of wave packet of scattering system, since harmonic approximation, that exactly propagates Gaussian wave packets, is a natural starting point of that theory [7].

The suggested development covers also two of the principle questions of the quantum theory. First one is connected to the possibility of study of the quantum system behavior in the region of close coupling. For that purpose it is necessary to build up a perturbation theory, that do not need a knowledge of the spectrum of unperturbed system. Also there are several such approaches (see i.e. [3]), they are not generalized to nonstationary case and so are not suited for scattering problems. Our method exactly serves for that purpose.

Second question is closely connected to the limits of quasiclassical description of the quantum system evolution. The traditional WKB approach, as it is well known, is limited for scattering applications. At the same time there is famous path integral approach that makes it possible to describe exactly quantum dynamics in terms of classical trajectories. So the basic question is how to improve the WKB approximation to describe all the effects, for which traditional approach fails. Our investigations makes it possible to develope the representation of the scattering theory via the classical trajectories and so build up the intermediate approximate solutions between exact and primitive WKB forms.

It is well known, that nonstationary form of the equation for quantum oscillator by a special transformations can be reduced to stationary form [10], that can be solved exactly. In our case unfortunately the use of such approach is not very effective, sine after such transformation the perurbation becomes so complicated, that analytic computation of the perturbation matrix elements would be unrealistic.

\section{Formulation of the problem}

Let us consider the Schroedinger equation for one-dimensional nonstationary anharmonic oscillator

$$
\begin{gathered}
\hat{L} \Psi+\lambda V(x, \tau) \Psi=0, \quad-\infty<x, \tau<+\infty \\
\stackrel{\wedge}{L}=i \partial_{\tau}+\frac{1}{2} \partial_{x}^{2}-\left(\frac{1}{2} \Omega^{2}(\tau) x^{2}-F(\tau) x\right)
\end{gathered}
$$


with $\Psi$ being the oscillator wave function, $\lambda$ being the dimensionless coupling parameter, and the perturbation potential $V(x, \tau)$, oscillator frequency $\Omega(\tau)$ and the external force $F(\tau)$ having the following asymptotic behavior

$$
V_{ \pm}(x)=\lim _{\tau \rightarrow \pm \infty} V(x, \tau), \quad \Omega_{\text {in }(\text { out })}=\lim _{\tau \rightarrow \pm \infty} \Omega(\tau), \quad \lim _{\tau \rightarrow \pm \infty} F(\tau)=0 .
$$

Let us assume, that solution of (2.1) satisfies the following boundary and initial conditions

$$
\begin{gathered}
\lim _{x \rightarrow \pm \infty} \Psi(x, \tau)=0, \quad \lim _{x \rightarrow \pm \infty} \Psi(x, \tau) \partial_{x} \Psi(x, \tau)=0, \\
\lim _{\tau \rightarrow-\infty} \Psi(x, \tau)=\Psi_{i n}\left(n ; \Omega_{i n}, x\right) \exp \left[-i\left(n+\frac{1}{2}\right) \Omega_{i n} \tau\right],
\end{gathered}
$$

were $\Psi_{i n}\left(n ; \Omega_{i n}, x\right)$ is a function of stationary anharmonic oscillator of initial channel.

The physical meaning of those conditions is obvious - the decay of probability and its flow at infinity - and it corresponds to the formulation of the physical problems, discussed in Introduction. When perturbation is absent, i.e. with $V=0$, the equation

$$
\hat{L} \Psi_{0}(n ; x, \tau)=0,
$$

with boundary conditions (2.3), (2.4) has an exact solution (see i.e. [8] )

$$
\begin{gathered}
\Psi_{0}(n ; x, \tau)=f_{0}(n ; x, \tau)=K(n ; \tau) \exp \left(a_{1}(\tau) y+a_{2}(\tau) y^{2}\right) H_{n}(y), \\
K(n ; \tau)=\left(\frac{\left(\Omega_{i n} / \pi\right)^{1 / 2}}{2^{n} n !|\zeta(\tau)|}\right)^{1 / 2} \exp \left(i \int_{-\infty}^{\tau} L_{c l}(\tau) d \tau-i \int_{-\infty}^{\tau} E(n ; \tau) d \tau\right), \\
a_{1}(\tau)=i \frac{\dot{\eta}|\zeta|}{\left(\Omega_{i n}\right)^{1 / 2}}, \quad a_{2}(\tau)=\frac{1}{2}\left(i \frac{|\zeta||\dot{\zeta}|}{\Omega_{i n}}-1\right), \quad \dot{\eta}=d \eta / d \tau \\
y=\left(\Omega_{i n}\right)^{1 / 2} \frac{x-\eta(\tau)}{|\zeta(\tau)|}, \quad|\dot{\zeta}|=\frac{d|\zeta|}{d \tau} \\
L_{c l}=\frac{1}{2}(\dot{\eta})^{2}-\frac{1}{2} \Omega^{2} \eta^{2}+F \eta, \quad E(n ; \tau)=\frac{\Omega_{i n}}{|\zeta(\tau)|^{2}}(n+1 / 2),
\end{gathered}
$$

with constant $\Omega_{i n}$ being the initial frequency, $n$ being the principle quantum number, $E(n ; \tau)$ being the adiabatically changing $n$-the energy level and the functions $\zeta(\tau), \eta(\tau)$ satisfy the equations

$$
\ddot{\zeta}+\Omega^{2}(\tau) \zeta=0, \quad \ddot{\eta}+\Omega^{2}(\tau) \eta=F(\tau),
$$

with following asymptotic and initial conditions

$$
\begin{gathered}
\zeta_{+}(\tau)=\lim _{\tau \rightarrow \infty} \zeta(\tau)=c_{1} \exp \left(i \Omega_{\text {out }} \tau\right)-c_{2} \exp \left(-i \Omega_{\text {out }} \tau\right), \\
\zeta_{-}(\tau)=\lim _{\tau \rightarrow-\infty} \zeta(\tau)=\exp \left(i \Omega_{\text {in }} \tau\right), \quad\left|c_{1}\right|^{2}-\left|c_{2}\right|^{2}=1, \\
\eta(-\infty)=\dot{\eta}(-\infty)=0 .
\end{gathered}
$$


It is well known, that solution of the second equation (2.8) can be constructed on the base of solution of corresponding homogeneous equation

$$
\eta(\tau)=\frac{1}{\sqrt{2 \Omega_{\text {in }}}}\left[\zeta(\tau) d^{*}(\tau)+\zeta^{*}(\tau) d(\tau)\right], \quad d(\tau)=\frac{i}{\sqrt{\Omega_{\text {in }}}} \int_{-\infty}^{\tau} d \tau^{\prime} \zeta\left(\tau^{\prime}\right) F\left(\tau^{\prime}\right)
$$

Semiclassical type analysis gives the following form of solution of (2.1)

$$
\Psi^{(+)}(n ; x, \tau)=f(n ; x, \tau) \exp (-\Phi(n ; x, \tau)),
$$

with $\Psi^{(+)}$being the total wavefunction, that is being developed from the $n$-the asymptotic excited state at $\tau \rightarrow-\infty$.

Substitution of (2.11) into (2.1) gives the following equation for unknown functions $\Phi$ and $f$

$$
\left(i \partial_{\tau} \Phi-\frac{1}{2}\left(\partial_{x} \Phi\right)^{2}+\frac{1}{2} \partial_{x}^{2} \Phi-\lambda V(x, \tau)\right) f-\left(\hat{L} f-\left(\partial_{x} \Phi\right)\left(\partial_{x} f\right)\right)=0
$$

For further investigation of equation (2.12) it is convenient to expand $f(n ; x, \tau)$ and $\Phi(n ; x, \tau)$ functions into power series over $\lambda$

$$
\begin{gathered}
\Phi(n ; x, \tau)=\sum_{k=0}^{\infty} \lambda^{k} \Phi_{k}(n ; x, \tau), \quad \Phi_{0}(n ; x, \tau)=0 \\
f(n ; x, \tau)=\sum_{k=0}^{\infty} \lambda^{k} f_{k}(n ; x, \tau)
\end{gathered}
$$

As in all exponential approximation approaches one term of expansion of $\Phi(n ; x, \tau)$ takes into account infinite number of terms of standard perturbation theory. But the more we try to take into account in the exponential, the more difficult would be the equations for individual members. In our case there is an optimal choice, that makes it possible to formulate additional condition for $(2.13),(2.14)$, and so to determine both functions $\Phi$ and $f$ in (2.11) without ambiguity. Let us start from the equation for " $k$ "-the correction

$$
i \partial_{\tau} \Phi_{k}+\frac{1}{2} \partial_{x}^{2} \Phi_{k}+q_{k}^{(1)}(x, \tau)-f_{0}^{-1}\left(\hat{L} f_{k}-\left(\partial_{x} \Phi_{k}\right)\left(\partial_{x} f_{0}\right)+q_{k}^{(2)}(x, \tau)\right)=0
$$

with

$$
\begin{gathered}
q_{k}^{(1)}=-\frac{1}{2} \sum_{m=1}^{k-1}\left(\partial_{x} \Phi_{m}\right)\left(\partial_{x} \Phi_{k-m}\right), \quad k \geq 2 \\
q_{k}^{(2)}(x, \tau)=-\sum_{m=1}^{k-1}\left[f _ { m } \left(i \partial_{\tau} \Phi_{k-m}+\frac{1}{2} \partial_{x}^{2} \Phi_{k-m}-\delta_{k-m, 1} V-\right.\right. \\
\left.\left.-\frac{1}{2} \sum_{l=1}^{k-m-1}\left(\partial_{x} \Phi_{l}\right)\left(\partial_{x} \Phi_{k-m-l}\right)\right)-\left(\partial_{x} \Phi_{m}\right)\left(\partial_{x} f_{k-m}\right)\right], \quad k \geq 2 .
\end{gathered}
$$

In case of $k=1$ one has simple relations 


$$
q_{1}^{(1)}(x, \tau)=-V(x, \tau), \quad q_{1}^{(2)}(x, \tau)=0 .
$$

Note, that the eigenenergies are not preserved in our problem and so the corrections to them in general give no useful information about the system. Thus the problem is reduced to the solution of equation (2.15) i.e. to determination of correction to wavefunction of anharmonic oscillator.

\section{Construction of the solution}

Before solving the equation (2.15) let us note two important points

a) The equation is not correctly formulated, as there are two unknown functions in one equation,

b) There is, in general, at least one singular member in this equation. It can be explained most easily from analysis of (2.16), taking into account, that $f_{0}(n ; x, \tau)$ includes the Hermitian polynomial. This difficulty is readily solved if the perturbation is of polynomial form, as we would suppose in future.

Such form of perturbation does not seriously influence our discussion, as any smooth perturbation can be approximated by a finite part of its Taylor expansion.

As in every exponential perturbation theory one can solve both above problems by choosing additional condition for equation (2.15). Let us start from equations of the first order in perturbation. It is convenient to choose

$$
\begin{gathered}
i \partial_{\tau} \Phi_{1}+\frac{1}{2} \partial_{x}^{2} \Phi_{1}+q_{1}^{(1)}(n ; x, \tau)+Q_{1}(n ; x, \tau)=0 \\
\stackrel{\wedge}{L} f_{1}=\left(\partial_{x} \Phi_{1}\right)\left(\partial_{x} f_{0}\right)-Q_{1}(n ; x, \tau) f_{0}
\end{gathered}
$$

with $Q_{1}(n ; x, \tau)$ being yet unknown function. With additional condition of $\Phi_{1}(n ; x, \tau)$ being nonsingular correction, one has that $\Phi_{1}(n ; x, \tau), Q_{1}(n ; x, \tau)$ and all the terms in (3.2) are polynomials of the same order, as $q_{1}^{(1)}(n ; x, \tau)$. By expanding the functions of eq. (3.2) in a series of Hermitian polynomials and supposing the absence of the members of an order, higher than $n$, one can correctly determine the coefficients of polynomial $Q_{1}(n ; x, \tau)$. So, it is clear that both above difficulties are thus overcome and the system of equations (3.1), (3.2) is quite correct. The procedure is readily generalized to any order. As for $k \geq 2 q_{k}^{(2)}(n ; x, \tau) \neq 0$, so one has

$$
\begin{gathered}
Q_{k}(n ; x, \tau)=\left\{\left(\partial_{x} \Phi_{k}\right)\left(\partial_{x} f_{0}\right)-\hat{L} f_{k}-\sum_{m=1}^{k-1}\left(f _ { m } \left[\left(i \partial_{\tau} \Phi_{k-m}\right)+\frac{1}{2} \partial_{x}^{2} \Phi_{k-m}-\right.\right.\right. \\
\left.\left.\left.-\delta_{k-m, 1} V-\frac{1}{2} \sum_{l=1}^{k-m-1}\left(\partial_{x} \Phi_{l}\right)\left(\partial_{x} \Phi_{k-m-l}\right)\right]-\left(\partial_{x} f_{k-m}\right)\left(\partial_{x} \Phi_{m}\right)\right)\right\} f_{0}^{-1} .
\end{gathered}
$$

Taking into account, that expression in square brackets is equal to $Q_{k-l}$, eq. (3.3) is written in the following form

$$
Q_{k}(n ; x, \tau)=\left\{\left(\partial_{x} \Phi_{k}\right)\left(\partial_{x} f_{0}\right)-\hat{L} f_{k}-\sum_{m=1}^{k-1}\left(f_{m} Q_{k-m}-\left(\partial_{x} f_{m}\right)\left(\partial_{x} \Phi_{k-m}\right)\right)\right\} f_{0}^{-1}
$$


and for determination of $f_{k}$ one has the equation

$$
\hat{L} f_{k}=\sum_{m=1}^{k-1}\left(\left(\partial_{x} f_{m}\right)\left(\partial_{x} \Phi_{k-m}\right)-f_{m} Q_{k-m}\right) .
$$

And again if one supposes, that $f_{m}, \Phi_{m}$ and $Q_{m}$ for $m \leq k$ are polynomials, then $Q_{k}$ and $f_{k}$ are easily determined and it is possible to find the equation for $\Phi_{k}$

$$
i \partial_{\tau} \Phi_{k}+\frac{1}{2} \partial_{x}^{2} \Phi_{k}+q_{k}^{(1)}(n ; x, \tau)+Q_{k}(n ; x, \tau)=0 .
$$

Note, that for polynomial $Q_{k}(n ; x, \tau)$ the solution of (3.6) is also polynomial and only high order terms of perturbation potential $V(x, \tau)$ are important for determination of $\Phi_{m}$ and $f_{m}$.

\section{Calculation of wavefunction to the first order of perturbation theory}

Let us study in more detail the case of asymmetric polynomial perturbation

$$
V(x, \tau)=\alpha(\tau) x^{3}+\beta(\tau) x^{4}=\sum_{m=0}^{4} b_{m}(\tau) y^{m},
$$

with coefficients $b_{m}(\tau)$ of the form

$$
\begin{gathered}
b_{0}(\tau)=\eta^{3}(\beta \eta+\alpha), b_{1}(\tau)=\Omega_{i n}^{-1 / 2} \eta^{2}|\zeta|(4 \beta \eta+3 \alpha), \\
b_{2}(\tau)=3 \Omega_{i n}^{-1} \eta|\zeta|^{2}(2 \beta \eta+\alpha), b_{3}(\tau)=\Omega_{i n}^{-3 / 2}|\zeta|^{3}(4 \beta \eta+\alpha), \\
b_{4}(\tau)=\Omega_{\text {in }}^{-2} \beta|\zeta|^{4}
\end{gathered}
$$

and assumption, that $\alpha(\tau)$ and $\beta(\tau)$ are slowly varying functions of $\tau$.

Before passing to the solution of (3.1) , (3.2) in accordance to previous discussion let us rewrite $\Phi_{1}$ and $Q_{1}$ in the following form

$$
\Phi_{1}(n ; x, \tau)=\sum_{k=0}^{4} v_{k}(n ; \tau) y^{k}, Q_{1}(n ; x, \tau)=\sum_{k=0}^{4} \sigma_{k}(n ; \tau) y^{k} .
$$

Thus, the additional condition, introduced for regularization of perturbation approach, can be expressed in the form

$$
\begin{gathered}
f_{0}(n ; x, \tau) \sum_{k=0}^{4} \sigma_{k}(\tau) y^{k}=\kappa_{0}\left(\left(2 a_{2}(\tau) y+a_{1}(\tau)\right) f_{0}(n ; x, \tau)+2 n K(n ; \tau) \times\right. \\
\left.\times K^{-1}(n-1 ; \tau) f_{0}(n-1 ; x, \tau)\right) \sum_{k=1}^{4} k v_{k}(\tau) y^{k-1}-\hat{L} f_{1}, \quad \kappa_{0}=\frac{\Omega_{i n}}{|\zeta(\tau)|^{2}}
\end{gathered}
$$

Writing down $f_{0}(n ; x, \tau)$ in explicit form and equating on both sides of $(4.4)$ coefficients of the polynomials of the same order we may determine the coefficients in polynomial $Q_{1}(n ; x, \tau)$ : 


$$
\begin{gathered}
\sigma_{0}(n ; \tau)=\kappa_{0}\left(a_{1} v_{1}+2 n v_{2}+2 n(n-1) v_{4}\right), \quad \sigma_{1}(n ; \tau)=2 \kappa_{0}\left(a_{2} v_{1}+a_{1} v_{2}+\frac{3}{2} n v_{3}\right), \\
\sigma_{2}(n ; \tau)=4 \kappa_{0}\left(a_{2} v+\frac{3}{4} a_{1} v_{3}+n v_{4}\right), \quad \sigma_{3}(n ; \tau)=6 \kappa_{0}\left(a_{2} v_{3}+\frac{2}{3} a_{1} v_{4}\right), \\
\sigma_{4}(n ; \tau)=8 \kappa_{0} a_{2} v_{4} .
\end{gathered}
$$

Substituting (4.5) in (3.1) one gets a system of nonuniform linear differential equations for determination of coefficients in the correction $\Phi_{1}(n ; x, \tau)$ :

$$
i \dot{v}_{j}-c_{j}(\tau) v_{j}-d_{j}(\tau)=0, \quad \dot{v}_{j}=d v_{j}(\tau) / d \tau, \quad j=0,1,2,3,4 .
$$

with functions $c_{j}(\tau)$ and $d_{j}(\tau)$ given by

$$
\begin{gathered}
c_{4}(\tau)=4 \kappa_{0}, \quad c_{3}(\tau)=3 \kappa_{0}, \quad c_{2}(\tau)=2 \kappa_{0}, \quad c_{1}(\tau)=\kappa_{0}, \quad c_{0}(\tau)=0, \\
d_{4}(\tau)=b_{4}, \quad d_{3}(\tau)=b_{3}, \quad d_{2}(\tau)=b_{2}+2(2 n+3) \kappa_{0} v_{4}, \\
d_{1}(\tau)=b_{1}+3(n+1) \kappa_{0} v_{3}, \quad d_{0}(\tau)=b_{0}+(2 n+1) \kappa_{0} v_{2}+2 n(n-1) \kappa_{0} v_{4} .
\end{gathered}
$$

Initial conditions for the system (4.5) are

$$
v_{0}^{-}=0, \dot{v}_{0}^{-}=-i d_{0}, v_{j}^{-}=-\frac{d_{j}^{-}}{c_{j}^{-}}, \quad j=1,2,3,4 .
$$

Note, that "-" parameters correspond to the limit $\tau \rightarrow-\infty$. It is clear that solution of (4.6) must start from $j=4$. In such a way, solution of each equation is presented in the following form

$$
v_{j}(\tau)=G_{j}(\tau,-\infty)\left[v_{j}^{-}-i \int_{-\infty}^{\tau} G_{j}\left(-\infty, \tau^{\prime}\right) d_{j}\left(\tau^{\prime}\right) d \tau^{\prime}\right], \quad j=0, \ldots, 4
$$

with $G_{j}\left(\tau, \tau^{\prime}\right)$ being the evolution operator of pertinent homogeneous equation

$$
G_{j}\left(\tau, \tau^{\prime}\right)=G_{j}^{-1}\left(\tau^{\prime}, \tau\right)=\exp \left[-i j \int_{\tau^{\prime}}^{\tau} \kappa_{0}\left(\tau^{\prime \prime}\right) d \tau^{\prime \prime}\right]
$$

Taking into account conditions (4.5) equation (4.4) can be represented in the following form

$$
\hat{L} f_{1}(n ; x, \tau)-\sum_{j=1}^{4} \bar{e}_{j}(n ; \tau) f_{0}(n-j ; x, \tau)=0
$$

with

$$
\bar{e}_{4}(n ; \tau)=2 \kappa_{0} v_{4}\left[\frac{n !}{(n-4) !}\right]^{1 / 2} G_{4}(-\infty, \tau)
$$




$$
\begin{gathered}
\bar{e}_{3}(n ; \tau)=3 \kappa_{0} v_{3}\left[\frac{n !}{2(n-3) !}\right]^{1 / 2} G_{3}(-\infty, \tau), \\
\bar{e}_{2}=\kappa_{0}\left[2(2 n-3) v_{4}+v_{2}\right]\left[\frac{n !}{(n-2) !}\right]^{1 / 2} G_{2}(-\infty, \tau), \\
\bar{e}_{1}(n ; \tau)=\kappa_{0}\left[3(n-1) v_{3}+2 v_{1}\right]\left[\frac{n !}{2(n-1) !}\right]^{1 / 2} G_{1}(-\infty, \tau) .
\end{gathered}
$$

In all cases, when $n-j \prec 0, \bar{e}_{j}$ are equal to zero. The solution of (4.11) is naturally represented in such a way

$$
f_{1}(n ; x, \tau)=\sum_{j=1}^{4} \bar{w}_{j}(n ; \tau) f_{0}(n-j ; x, \tau), \bar{w}_{j}(n ; \tau)=G_{j}(-\infty, \tau) w_{j}(n ; \tau)
$$

Substituting (4.13) into (4.11) one gets the linear first-order equations for coefficients $w_{j}(\tau)$

$$
i \dot{w}_{j}-j \kappa_{0}(\tau) w_{j}-e_{j}(n ; \tau)=0, e_{j}(n ; \tau)=\bar{e}_{j}(n ; \tau) G_{j}(\tau,-\infty),
$$

with initial conditions

$$
w_{j}^{-}=\lim _{\tau \rightarrow-\infty} w_{j}(n ; \tau)=-\frac{e_{j}(n,-\infty)}{j \kappa_{0}(-\infty)}, \quad j=1, \ldots, 4 .
$$

Solution of this equation is obvious,

$$
w_{j}(\tau)=G_{j}(\tau,-\infty)\left[w_{j}^{-}-i \int_{-\infty}^{\tau} d \tau^{\prime} G_{j}\left(-\infty, \tau^{\prime}\right) e_{j}\left(\tau^{\prime}\right)\right] .
$$

Solutions for higher orders of expansion are constructed in the same way.

\section{Calculation of the transition $S$-matrix for nonstationary an- harmonic oscillator}

The fact, that unharmonic oscillator wave functions, determined above, are nonstationary, opens new possibilities in view of our previous result [6]. It was shown there, that scattering in three-body collinear system is effectively reduced to the evolution of anharmonic oscillator in external field. And so the above wave functions can be used for computation of the scattering matrix. It is not evident how to calculate the scattering matrix elements via the oscillator functions. It is possible to show, using the development of [5], that $S$-matrix elements for scattering problems can be represented by one-dimensional integral in the form, similar to standard representation [9] of nonstationary $S$-matrix:

$$
S_{m n}=\lim _{\tau \rightarrow+\infty}\left\langle\psi_{f}^{*}(m ; x, \tau) \psi^{+}(n ; x, \tau)\right\rangle, \quad\langle\ldots\rangle=\int_{-\infty}^{\infty} \ldots d x
$$


with $\psi_{f}(m ; x, \tau)$ being the asymptotic wave function of final state. Let us have a look at the approximations of exact and asymptotic wave functions. For nonstationary wave function $\psi^{(+)}(n ; x, \tau)$ with the help of (2.11) to the first order of perturbation theory over $\lambda$ one has

$$
\begin{aligned}
& \psi^{(+)}(n ; x, \tau)=\left[f_{\lambda}(n ; x, \tau)+\lambda f_{\lambda}^{1}(n ; x, \tau)\right] \exp \left[-\lambda \sum_{l=1}^{4} v_{l}(\tau) y^{l}\right]+O\left(\lambda^{2}\right), \\
& f_{\lambda}(n ; x, \tau)=\exp \left[-\lambda v_{0}(\tau)\right] f_{0}(n ; x, \tau), f_{\lambda}^{1}(n ; x, \tau)=\exp \left[-\lambda v_{0}(\tau)\right] f_{1}(n ; x, \tau) .
\end{aligned}
$$

Note, that functions $f_{0}(n ; x, \tau)$ and $f_{1}(n ; x, \tau)$ are determined by the formulae $(2.6)-$ (2.7) and (4.13). Expanding the exponential function in (5.2) one has

$$
\psi^{(+)}(n ; x, \tau)=f_{\lambda}(n ; x, \tau)+\lambda\left[f_{\lambda}^{1}(n ; x, \tau)-f_{\lambda}(n ; x, \tau) \sum_{l=1}^{4} v_{l}(\tau) y^{l}\right]+O\left(\lambda^{2}\right) .
$$

Now one can use well known formula for Hermitian polynomials $x H_{m}(x)=1 / 2 H_{m+1}(x)+$ $m H_{m-1}(x)$ and expand in (5.3) the expressions of type $y^{j} f_{\lambda}(n ; x, \tau)$ into the series over the Hermitian polynomials. So one gets

$$
\begin{gathered}
\psi^{(+)}(n ; x, \tau)=f_{\lambda}(n ; x, \tau)+ \\
+\lambda\left[\sum_{j=1}^{4} \bar{w}_{l}(n ; \tau) f_{\lambda}(n-l ; x, \tau)-\sum_{p=-4}^{4} \bar{u}_{p}(n ; \tau) f_{\lambda}(n-p ; x, \tau)\right]+O\left(\lambda^{2}\right),
\end{gathered}
$$

with

$$
\bar{u}_{p}(n ; \tau)=G_{p}(\tau,-\infty) u_{p}(n ; \tau), \quad u_{p}(n ; \tau)=\left[\frac{(n-p) !}{2^{p} n !}\right]^{1 / 2} \chi_{p}(n ; \tau) .
$$

As to $\chi_{p}(n ; \tau)$ functions, they are given by the following expressions

$$
\begin{gathered}
\chi_{-4}(n ; \tau)=\frac{1}{2^{4}} v_{4}(\tau), \quad \chi_{-3}(n ; \tau)=\frac{1}{2^{3}} v_{3}(\tau) \\
\chi_{-2}(n ; \tau)=\frac{1}{2}\left(n+\frac{3}{2}\right) v_{4}(\tau)+\frac{1}{2^{2}} v_{2}(\tau), \quad \chi_{-1}(n ; \tau)=\frac{3}{4}(n+1) v_{3}(\tau)+\frac{1}{2} v_{1}(\tau) \\
\chi_{0}(n ; \tau)=\frac{3}{2}\left(n^{2}+n+\frac{1}{2}\right) v_{4}(\tau)+\left(n+\frac{1}{2}\right) v_{2}(\tau) \\
\chi_{4}(n ; \tau)=n(n-1)(n-2)(n-3) v_{4}(\tau), \quad \chi_{3}(n ; \tau)=n(n-1)(n-2) v_{3}(\tau) \\
\chi_{2}(n ; \tau)=3 n(n-1)^{2} v_{4}(\tau)+n(n-1) v_{2}(\tau), \quad \chi_{1}(n ; \tau)=\frac{3}{2} n^{2} v_{3}(\tau)+n v_{1}(\tau)
\end{gathered}
$$


In the same way we can get the representations for asymptotic states of anharmonic oscillator. So for (out) state one has

$$
\begin{gathered}
\psi_{f}(m ; x, \tau)=\varphi_{f}^{0}(m ; x, \tau)+ \\
+\lambda\left[\sum_{l=1}^{4} \bar{w}_{l}^{f}(m ; \tau) \varphi_{f}^{0}(m-l ; x, \tau)-\sum_{p=-4}^{4} \bar{u}_{p}^{f}(m ; \tau) \varphi_{f}^{0}(m-p ; x, \tau)\right]+O\left(\lambda^{2}\right) .
\end{gathered}
$$

Function $\varphi_{f}^{0}(m ; x, \tau)$ in the above expression is an (out) asymptotic state of harmonic oscillator

$$
\begin{gathered}
\varphi_{f}^{0}(m ; x, \tau)=\varphi_{f}^{0}\left(m ; \Omega_{\text {out }} ; x\right) \exp \left[-i\left(m+\frac{1}{2}\right) \Omega_{\text {out }} \tau\right], \\
\varphi_{f}^{0}(m ; \Omega ; x)=\left[\frac{(\Omega / \pi)^{1 / 2}}{2^{m} m !}\right]^{1 / 2} \exp \left(-\frac{1}{2} \Omega x^{2}\right) H_{m}(\sqrt{\Omega} x) .
\end{gathered}
$$

In the above expressions $\bar{w}_{l}^{f}(m ; \tau)$ and $\bar{u}_{p}^{f}(m ; \tau)$ functions are given by

$$
\begin{gathered}
\bar{w}_{l}^{f}(m ; \tau)=w_{l}^{f}(m) \exp \left(-i l \Omega_{\text {out }} \tau\right), \\
\bar{u}_{p}^{f}(n ; \tau)=u_{p}^{f}(n) \exp \left(-i p \Omega_{\text {out }} \tau\right) .
\end{gathered}
$$

As to the coefficients $w_{l}^{f}(m)$ and $u_{p}^{f}(n)$, they can be obtained at once from (4.16) and (5.7) $-(5.8)$ in the limit $\tau \rightarrow-\infty$ after substitution $w_{j}^{-} \rightarrow w_{j}^{f}$ and $v_{j}^{-} \rightarrow v_{j}^{f}$. Now taking into account (5.5) and (5.10) and using (5.3) and (5.6) in (5.1) one gets

$$
S_{m n}(\lambda)=\left(S_{m n}^{0}+\lambda\left[S_{m n}^{1}+S_{m n}^{2}\right]\right) \exp \left[-\lambda v_{0}^{(+)}\right]+O\left(\lambda^{2}\right)
$$

with

$$
\begin{gathered}
S_{m n}^{1}=\sum_{l=1}^{4} w_{l}^{f}(m) S_{(m-l) n}^{0}-\sum_{p=-4}^{4} u_{p}^{f}(m) S_{(m-p) n}^{0}, \\
S_{m n}^{2}=\sum_{l=1}^{4} w_{l}^{(+)}(m) S_{m(n-l)}^{0}-\sum_{p=-4}^{4} u_{p}^{(+)}(n) S_{m(n-p)}^{0}, \\
S_{m n}^{0}=\lim _{\tau \rightarrow+\infty}\left\langle\left(\varphi_{f}^{0}(m ; x, \tau)\right)^{*} f_{0}(n ; x, \tau)\right\rangle .
\end{gathered}
$$

Now, starting from $(5.12)-(5.15)$, it is not difficult to get the analytic expressions for transition probabilities for anharmonic oscillator,

$$
\begin{gathered}
W_{m n}(\lambda)=\exp \left[-2 \lambda v_{0}^{(+)}\right]\left[1+2 \lambda \operatorname{Re}\left(\frac{S_{m n}^{1}+S_{m n}^{2}}{S_{m n}^{0}}\right)\right] W_{m n}^{0}+O\left(\lambda^{2}\right) \\
W_{m n}^{0}=\left|S_{m n}^{0}\right|^{2}=\lim _{\lambda \rightarrow 0} W_{m n}(\lambda) .
\end{gathered}
$$


In the expressions above, the value $S_{m n}^{0}$ is a matrix element of $S$-matrix for transitions in harmonic oscillator with variable frequency $\Omega(\tau)$ in the external field. The matrix element in (5.15) is calculated via the generating function [6] (see also [10]). So we can give here only the final result

$$
W_{m n}^{0}=\left(\frac{1-\rho}{m ! n !}\right)^{1 / 2}\left|H_{m n}\left(y_{1}, y_{2}\right)\right|^{2} \exp [-\nu(1-\sqrt{\rho} \cos 2 \theta)] .
$$

Here $H_{m n}\left(y_{1}, y_{2}\right)$ is the Hermitian polynomial of two variables [10] with

$$
y_{1}=\sqrt{\nu(1-\rho)} e^{i \theta}, \quad y_{2}=-\sqrt{\nu}\left(e^{-i \theta}-\sqrt{\rho} e^{i \theta}\right), \quad \theta=\frac{1}{2}\left(\delta_{1}+\delta_{2}\right)-\beta .
$$

$\nu, \rho, \delta_{j}$ and $\beta$ parameters are determined from solution of the classical problem for harmonic oscillator $(2.3),(2.9)$ and are given by following expressions:

$$
\begin{gathered}
c_{1}=e^{i \delta_{1}} \sqrt{\frac{\Omega_{\text {in }}}{\Omega_{\text {out }}}} \frac{1}{(1-\rho)^{1 / 2}}, \quad c_{2}=e^{i \delta_{2}} \sqrt{\frac{\Omega_{\text {in }}}{\Omega_{\text {out }}}}\left(\frac{\rho}{1-\rho}\right)^{1 / 2}, \\
\rho=\left|\frac{c_{2}}{c_{1}}\right|^{2}, \quad d=\lim _{\tau \rightarrow+\infty} d(\tau)=\sqrt{\nu} e^{i \beta} .
\end{gathered}
$$

Note, that in the expression proposed both for $S$-matrix (5.1) and for transition probability $W_{m n}$ (5.16) we used only the first members of the expansion of exponentials in Taylor series over coordinate $y$. Such approximations for wave function and transition probability are effectively used for many interesting applications. In some cases, when anharmonic perturbation substantially change spectrum of the problem and thus our expansion bases, the leading terms of perturbation must be taken into account.

It is clear, that for scattering problems unharmonic perturbation is very important for higher excited states, especially for rearrangement procceses. At the same time in some situations for rearrangement processes even for ground state the effect of perturbation can be substantial. To show it let us discuss one particular case.

\section{Application to "ground state-ground state" transition}

We shall demonstrate an application of the method proposed to most simple situation - the perturbation of parametric harmonic oscillator by symmetric potential $\beta(\tau) x^{4}$, with $\beta(\tau)$ being adiabatically changing functions with boundary values $\beta(-\infty)=0$ and $\beta(\infty)=$ $\beta^{+} \neq 0$. Note, that transition probability for unperturbed oscillator can be obtained

from (5.16) with $\nu=0$, or by Taylor expansion of generating function of corresponding $S$-matrix [10]

$$
\begin{gathered}
W_{m n}^{(0)}=\left|S_{m n}^{(0)}\right|^{2}=\frac{n_{<} !}{n_{>} !} \sqrt{1-\rho}\left|P_{\left(\begin{array}{c}
\left.n_{>}-n_{<}\right) / 2 \\
\left(n_{<}\right) / 2
\end{array}\right.}^{(\sqrt{1-\rho})}\right|^{2} \\
S_{m n}^{(0)}(\rho)=\frac{1}{\sqrt{m ! n !}}\left\{\partial_{z_{1}}^{m} \partial_{z_{2}}^{n} I\left(z_{1}, z_{2} ; \rho\right)\right\}_{z_{1}=z_{2}=0},
\end{gathered}
$$

were generating function $I$ is equal 


$$
I\left(z_{1}, z_{2} ; \rho\right)=(1-\rho)^{1 / 4} \exp \left\{\frac{1}{2}\left[\sqrt{\rho}\left(z_{1}^{2}-z_{2}^{2}\right)+2 \sqrt{1-\rho} z_{1} z_{2}\right]\right\},
$$

with $n_{<}=\min (m, n), n_{>}=\max (m, n)$, and $P_{n}^{m}(x)$ being the associated Legendre polynomial.

For many reasons one of most important parameters of the problem is $W_{00}(\lambda ; \rho)$, that measures the probability of change of initial ground state to final ground state. So we shall discuss it in some details. From $(6.1)-(6.3)$ one gets

$$
\begin{gathered}
W_{00}(\lambda ; \rho)=\exp \left[-\lambda v_{0}^{(+)}\right][1-2 \operatorname{Re} \Lambda(\rho)] W_{00}^{(0)}(\rho), \\
\Lambda(\rho)=\left[S_{00}^{(0)}\right]^{-1} \sum_{k=0}^{2}\left(u_{-2 k}^{f} S_{2 k, 0}^{(0)}+u_{-2 k}^{(+)} S_{0,2 k}^{(0)}\right),
\end{gathered}
$$

with matrix elements $S_{2 k, 0}^{(0)}$ and $S_{02, k}^{(0)}$ given by $(6.2)-(6.3)$,

$$
S_{00}^{(0)}=(1-\rho)^{1 / 4}, \quad S_{20}^{(0)}=-S_{02}^{(0)}=\frac{1}{\sqrt{2 !}} \sqrt{\rho} S_{00}^{(0)}, \quad S_{40}^{(0)}=S_{04}^{(0)}=\frac{1}{\sqrt{4 !}} \rho S_{00}^{(0)}
$$

For determination of $u_{-2 k}^{f}$ and $u_{-2 k}^{(+)}$coefficients, that are important for $\Lambda(\rho)$ dependence and so the dependence of scattering amplitude, one must find coefficients $v_{j}^{f}$ and $v_{j}^{(+)}=v_{j}(\infty)$. After integration of (4.9) by parts and taking into account adiabatic dependence $\beta(\tau)$ one gets

$$
v_{j} \simeq-\frac{d_{j}}{j k_{0}(\tau)}+\frac{1}{j} G_{j}(\tau ;-\infty) \int_{-\infty}^{\tau} d \tau^{\prime} G_{j}\left(-\infty, \tau^{\prime}\right) \frac{\dot{d}_{j}\left(\tau^{\prime}\right)}{k_{0}\left(\tau^{\prime}\right)}, \quad j=1,2,3,4
$$

Now the coefficients $v_{j}^{(+)}$are determined from (6.7) taking into account (4.7) - (4.8) via integration by parts and averaging over fast oscillations

$$
\begin{gathered}
v_{4}^{(+)} \simeq-\frac{\beta^{+}}{4 \bar{k}_{0}^{-3}}, \quad v_{2}^{(+)} \simeq \frac{3 \beta^{+}}{4 \bar{k}_{0}^{3}}, \quad v_{0}^{(+)} \simeq \frac{3 \beta^{+}}{8 \bar{k}_{0}^{3}}, \\
\bar{k}_{0}=\Omega_{i n} /\left(\left|c_{1}\right|^{2}+\left|c_{2}\right|^{2}\right) .
\end{gathered}
$$

In the same way, for coefficients $v_{j}^{f}$ one has

$$
\begin{gathered}
v_{4}^{f}=-\frac{d_{4}^{f}}{c_{4}^{f}}=-\frac{1}{4} \frac{\beta^{+}}{\Omega_{\text {out }}^{3}}, \quad v_{2}^{f}=-\frac{d_{2}^{f}}{c_{2}^{f}}=\frac{3}{4} \frac{\beta^{+}}{\Omega_{\text {out }}^{3}}, \quad v_{0}^{f}=0, \\
c_{j}^{f}=j \Omega_{\text {out }} .
\end{gathered}
$$

Now using $(5.5)-(5.7)$ from $(6.8)-(6.9)$ one gets the expressions for coefficients $u_{-2 k}^{+}$ and $u_{-2 k}^{f}$ in the form

$$
u_{0}^{f}=\frac{3}{16}\left(\frac{\beta^{+}}{\Omega_{\text {out }}^{3}}\right), \quad u_{0}^{+}=\frac{3}{16} \frac{\beta^{+}}{\Omega_{\text {out }}^{3}}\left(\frac{1+\rho}{1-\rho}\right)^{3},
$$




$$
u_{-2}^{f}=u_{-2}^{+}=0, \quad u_{-4}^{f}=-\frac{1}{4} \sqrt{\frac{3}{2}} \frac{\beta^{+}}{\Omega_{\text {out }}^{3}}, \quad u_{-4}^{+}=-\frac{1}{4} \sqrt{\frac{3}{2}} \frac{\beta^{+}}{\Omega_{\text {out }}^{3}}\left(\frac{1+\rho}{1-\rho}\right)^{3} .
$$

The formula (6.4) with the results above, gives the final expression for $W_{00}$

$$
W_{00}(\lambda ; \rho) \simeq \sqrt{1-\rho}\left\{1-\lambda\left[1-v_{0}^{+}(\rho)\right]\left(1-\frac{1}{3} \rho\right)\right\} \exp \left[-\lambda v_{0}^{+}(\rho)\right]
$$

with new notations

$$
v_{0}^{+}(\rho)=\frac{\Omega_{\text {out }}^{3}}{\beta^{+}} v_{0}^{(+)}=\left(\frac{1+\rho}{1-\rho}\right)^{3}, \quad \lambda \rightarrow \frac{3 \beta^{+}}{8 \Omega_{\text {out }}^{3}} \lambda .
$$

As it was shown in [10], parameter $\rho$, that measures the excitation of classical oscillator, corresponds to quantum mechanical refection coefficient of the particle with momentum $k(x)=\Omega(x)$. That makes it possible to use well known results from quantum mechanics for $\rho$.

As can be seen from fig.1 anharmonic oscillator unlike the harmonic parametric one in the limit $\rho \rightarrow 0$ have a transition probability non equal to one. More than that as it is seen from (6.11) the dependence of transition probability over $\lambda$ is regular, that makes it possible to use the proposed formula up to the values of $\lambda$ of the order of unity.

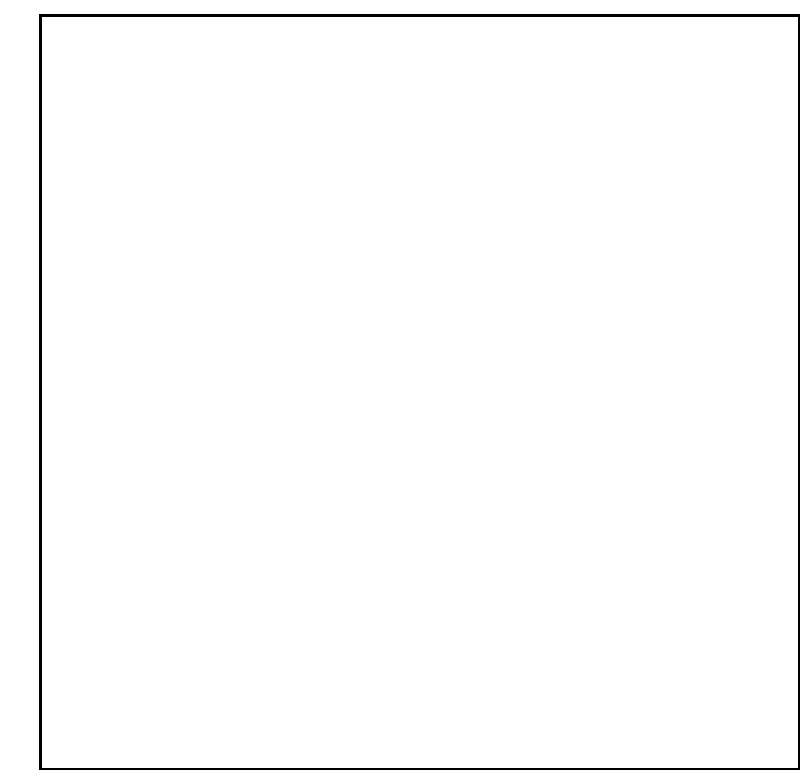

Fig. 1. The dependence of "ground state-ground state" transition probability over reflection coefficient $\rho$ and compliny constant $\lambda$.

\section{Conclusions}

Many important problems in theoretical and mathematical physics are reduced to the solution of the equation of nonstationary unharmonic oscillator with different sets of (in) and (out) states. The use of standard approaches to such problem meets two basic difficulties: 
a) Opposite to the stationary situation we do not have the fixed basic set for perturbed wave function;

b) In nonstationary situation the dimensionless perturbation parameter changes with time and can become not small in strong coupling region.

In this communication, as a generalization of nonlinearization method [3], we propose the way to overcome the above difficulties. The perturbation theory is constructed on exact wave functions for quantum harmonic oscillator with variable frequency in external field as basis state. This makes it possible, due to some unique intrinsic properties of those solutions, to work out the system of two linear equations, that determine the first corrections both to the amplitude and to the phase of total wave function. It should be noted also, that $n$-th order correction is also determined by two independent differential equations, that are obtained after $n$ iterations.

Very important computational property of our approach is connected to the fact, that any order correction is constructed on the bases of finite number of wave vectors. That makes it possible the analytical calculation of the corrections even to the transition operator.

The possibility of simultaneous correction of the phase and amplitude of the wave functions, as in a case of stationary problem, gives a regular method of investigation of strong coupling region, where perturbation is strong and small parameter is absent.

The approach is well suited for use in numerous application, of which we point out only some most important:

* corrections to path integral representation of the propagator in field and scattering theories,

* account of kinetics in some problems of solid state and condensed matter physics,

* anharmonic corrections for scattering matrix in collinear model of rearrangement collisions,

* anharmonic corrections for the propagator in the wave packet dynamics approach to molecular scattering.

We shall discuss those problems in detail in our future publications.

Note, that analysis of proposed expressions show, that account for antisymmetric terms in potential expansion can cause the nonadiabatic behavior and so nonanalytic dependence of $W_{00}(\lambda ; \rho)$ over $\lambda$. Depending on the form of perturbation "ground state ground state" transition probability might have in such a case several maxima.

Authors gratefully acknowledge the helpful discussions with Dr. Yu. Gorbachev.

\section{References}

[1] F.T. Hioe, D. McMillen, E.W. Montroll, Phys. Rep., 1978, v. 43, p. 306.

[2] M.I. Klinger, Phys., Rep., 1983, v. 94, p. 183.

[3] A.V. Turbiner, Sov. Jorn., Usp. Fiz. Nauk, 1984, v. 144, issue 1, pp. 79-112.

[4] A.D. Dolgov, A.V. Turbiner, Phys. Lett. Ser. A, 1980, v. 77, p. 15.

[5] A.S. Gevorkyan, Rep. NAS of Armenia, 1995, v. 95, N 3, pp. 146-151.

[6] A.V. Bogdanov, A.S. Gevorkyan and G.V. Dubrovskii, Pis'ma Zh. Tekh. Fiz., 1994, v. 20 , pp. $39-45$. 
[7] N.E. Henriksen, E.J. Heller, Chem. Phys. Lett., 1988. v. 148, N 6, pp. 567-571.

[8] V.S. Popov, A.M. Perelomov, Sov. Jorn. - Teor. Mat. Fiz., 1970, v.3, p. 377; Teor. Mat. Fiz., 1970, v. 4, p. 48.

[9] R.G. Newton - "Scattering theory of waves and particles", Mc Graw-Hill book company, N. York, 1966.

[10] A.I. Baz', Ya.B. Zel'dovich and A.M. Perelomov, Scattering reactions, and Decays in Nonrelativistic Quantum Mechanics [in Russian], Moscow, 1971. 
This figure "random.gif" is available in "gif" format from: http://arxiv.org/ps/quant-ph/9712057v1 